significant implications in the field for stimulating new ideas, both in the design of new ultrashort coherent lasers and in the comprehension of complex dynamics of light organization in systems characterized by many degrees of freedom.
Andrea Fratalocchi is in the Department of

Physics, Sapienza University of Rome, P.le A. Moro,

2 - 00185, Rome, Italy.

e-mail:andrea.fratalocchi@uniroma.it

References

1. http://nobelprize.org/nobel_prizes/physics/laureates/2001/ cornellwieman-lecture.pdf
2. Ketterle, W. Rev. Mod. Phys. 74, 1131-1151 (2002).

3. Connaughton, C. et al. Phys. Rev. Lett. 95, 263901 (2005).

4. Conti, C. et al. Phys. Rev. Lett. 101, 143901 (2008).

5. Weill, R., Fischer, B. \& Gat, O. Phys. Rev. Lett. 104, 173901 (2010).

6. Brabec, T. \& Krausz, F. Rev. Mod. Phys. 72, 545-591 (2000).

7. Hackenbroich, G., Viviescas, C. \& Haake, F. Phys. Rev. Lett. 89, 083902 (2002).

\title{
TERAHERTZ TECHNOLOGY
}

\section{Towards THz integrated photonics}

The demonstration of an integrated terahertz transceiver featuring a quantum cascade laser and a Schottky diode mixer promises new applications for compact and convenient terahertz photonic instrumentation.

\section{Heinz-Wilhelm Hübers}

T he terahertz (THz) spectral range, located between the infrared and millimetre-wavelength spectral regions, is one of the least-explored parts of the electromagnetic spectrum. Although it has interesting applications in astronomy, particularly for spaceborne $\mathrm{THz}$-sensing observatories such as the Herschel Space Observatory $^{1}$, the $\mathrm{THz}$ range still remains a rather exotic field. In the past few years there has been increasing attention on $\mathrm{THz}$ research and development, triggered by tremendous progress in the development of sources, detectors, optics and systems. $\mathrm{THz}$ technology is now on the verge of seeing commercial applications, for example in security, biomedicine, broadband communication, non-destructive testing and process control ${ }^{2}$.

An important technique in $\mathrm{THz}$ research, particularly for 'real-world' applications, is heterodyne detection. Widely used in radiofrequency telecommunications, heterodyne detection is popular because of its sensitivity, narrow bandwidth and ability to measure high-frequency signals, for which direct detection would otherwise prove challenging. The principle of heterodyne detection is that a signal carrying information is combined with radiation from a local oscillator operating at a frequency near that of the signal (Fig. 1a) using a component called a 'mixer'. The output from the mixer is a new signal that oscillates at a frequency called the beat frequency, which is equal to the frequency difference between the local oscillator and the original signal. The beat frequency is often easier to amplify and process than the original signal because it is at a much lower frequency.

In existing $\mathrm{THz}$ heterodyne receivers, the local oscillator and mixer are discrete units, making the whole system rather
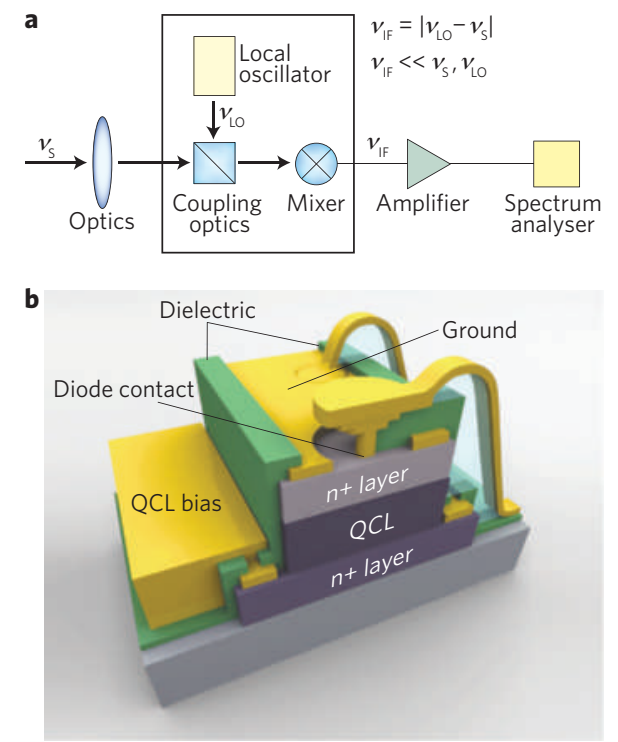

Figure 1 | Scheme of a heterodyne receiver and an integrated transceiver. $\mathbf{a}$, The principle of operation of a heterodyne receiver. $v_{\mathrm{LO}}$ is the frequency of the local oscillator, $v_{\mathrm{s}}$ is the frequency of the signal and $v_{\mathrm{IF}}$ is the beat frequency generated by the mixer. $v_{\mathrm{IF}}$, the difference between $v_{\mathrm{LO}}$ and $v_{\mathrm{S}}$, is often easier to amplify and process than $v_{\mathrm{S}}$ because it is at a much lower frequency. $\mathbf{b}$, Structure of the integrated transceiver of Wanke et al. The Schottky diode is on top of the QCL waveguide ridge. The integrated transceiver replaces the discrete local oscillator and mixer units as well as the coupling optics shown in a.

complex and thus limiting applications mostly to fields such as astronomy or Earth observation. Much current research focuses on developing discrete heterodyne receivers with quantum-noise-limited performance, which requires superconducting mixers to be cooled to around $4 \mathrm{~K}$. For cases in which cooling to such low temperatures is not an option, Schottky diode mixers can be used instead, but at the expense of lower sensitivity. In addition, heterodyne arrays with modest (less than 20) pixel numbers have also been realized, or are at least currently under development. However, all of these approaches rely on discrete mixer and local oscillator units. The realization of integrated circuits for performing various $\mathrm{THz}$ signal processing tasks is therefore highly desirable as it could ultimately allow the production of convenient, compact and potentially mass-producible low-cost equipment for $\mathrm{THz}$ applications.

Now, writing in Nature Photonics, Michael Wanke and co-workers from Sandia National Laboratories and LMATA Government Services in the USA report the first step towards this goal, with the demonstration of integrated circuitry for performing $\mathrm{THz}$ heterodyne detection ${ }^{3}$. The researchers have successfully demonstrated an integrated $\mathrm{THz}$ transceiver consisting of a $2.8 \mathrm{THz}$ quantum cascade laser (QCL) as a local oscillator and a Schottky barrier diode as a mixer. They show that this integrated circuit performs basic but important functions such as the transmission of a coherent carrier, heterodyne detection of an external signal, and frequency locking and tuning.

THz QCLs are typically based on a thin $(\sim 10 \mu \mathrm{m}) \mathrm{GaAs} / \mathrm{AlGaAs}$ superlattice on a semi-insulating GaAs substrate ${ }^{4}$. The superlattice is the active medium of the laser and has the form of a ridge a few millimetres long and 50-200 $\mu \mathrm{m}$ wide. On top of the ridge is a metal layer, and between the ridge and the substrate another metal layer or a highly doped GaAs layer is located. These layers form the waveguide of the QCL. The laser drive current is supplied through the 
top and bottom layers, and for continuouswave operation cooling to around $100 \mathrm{~K}$ or less is required. THz Schottky diode mixers are based on metal/GaAs interfaces; the GaAs layer must be highly doped (up to $10^{18} \mathrm{~cm}^{-3}$ ) and the contact area must be less than $1 \mu \mathrm{m}^{2}$ in size ${ }^{5}$.

QCLs and Schottky diode mixers are elaborate devices, which makes the fabrication of high-performance discrete devices a challenge - fabricating an integrated circuit that contains both is an even more difficult task. The approach realized by $\mathrm{M}$. Wanke et al. (Fig. 1b) relies on using an $\mathrm{n}^{+}$-doped GaAs layer to serve as both the top layer of the QCL superlattice and the cathode contact for the Schottky diode. In this case, the superlattice ridge of the QCL is $170 \mu \mathrm{m}$ wide and $3 \mathrm{~mm}$ long, with both end facets being uncoated to provide a Fabry-Pérot cavity with a longitudinal mode spacing of $13 \mathrm{GHz}$. A plasmonic waveguide with a metallic top layer and a GaAs layer between the active medium and the substrate confines the laser modes. The internal electric field of the waveguide is strongest near the top layer, which is desirable because the Schottky diode is implemented there. A less-doped 40-nm-thick $\mathrm{n}^{+}$-GaAs layer is then grown on top of the $n^{+}-G a A s$ layer of the QCL. In the centre of the top layer is an opening in the metal, in which a $1-\mu \mathrm{m}$ diameter metal contact is deposited - this is the anode of the Schottky diode. A big advantage of the integrated design is that the optical components for coupling the local oscillator radiation to the mixer and the need for optical alignment are eliminated.

The team demonstrated that their transceiver, mounted in a liquid helium flow cryostat and operated at a temperature near $10 \mathrm{~K}$, can perform the basic functions of a heterodyne receiver. Mixing the modes of the QCL in the Schottky diode resulted in a beat signal at the mode spacing frequency of $13 \mathrm{GHz}$, with a signal-to-noise ratio of $\sim 25 \mathrm{~dB}$. This beat signal was used as an active feedback signal for phase-locking the QCL to a microwave reference. Heterodyne detection was then demonstrated by mixing the radiation from an optically pumped $\mathrm{THz}$ gas laser with the modes of the QCL, with emission from the gas laser being focused onto one end facet of the QCL ridge. Part of the radiation was coupled into the waveguide ridge and mixed with the QCL modes in the Schottky diode, resulting in several beat signals within the $25 \mathrm{GHz}$ bandwidth of the detection system. The device was found to have a minimum detectable power of $0.1 \mu \mathrm{W}$ and a limited frequency tuning range of $1.6 \mathrm{GHz}$, induced by changing the temperature of the transceiver.

It is clear that the performance of the integrated transceiver is significantly worse than that of designs based on discrete mixers and local oscillator units. For example, the beat signal between longitudinal modes of a multimode QCL measured with a separate whisker-contacted Schottky diode has a signal-to-noise ratio of about $80 \mathrm{~dB}$ (ref. 6). Previous designs have attained sensitivities close to the quantum limit by using a superconducting hot-electron bolometric mixer and a QCL as a local oscillator such sensitivities are orders of magnitude below the minimum detectable power of the integrated transceiver ${ }^{7}$.

The integrated transceiver will require considerable development before it can become a competitive and widely usable device. In particular, improving the sensitivity of the Schottky diode is crucial. Optimization of the QCL is also an important future task. Ultimately, the size, mass, power consumption and complexity of the integrated transceiver will be constrained by its cooling requirements; lowering the electrical input power or increasing the operating temperature of the device, for example, will allow it to be mounted in a miniature cryocooler ${ }^{8}$. Another breakthrough would be to couple the external signal to the transceiver more efficiently, for example via some kind of antenna integrated with the Schottky diode rather than the coupling of radiation through the end facet of the QCL. Applications such as high-resolution spectroscopy will require a wider frequency tuning range and the stabilization of the QCL to an external reference. However, even with these improvements it might still be difficult to outperform heterodyne receivers based on discrete components.

Nevertheless, integrated transceivers may have important applications in the $\mathrm{THz}$ range as their shortcomings in sensitivity are compensated for by their low-complexity coupling optics and relative ease-ofintegration into large-format arrays. Given the broad range of possible applications it seems likely that integrated transceivers will have a significant role in the future scientific and commercial exploitation of the $\mathrm{THz}$ spectral range.

Heinz-Wilhelm Hübers is at the German Aerospace Center (DLR) and Technical University of Berlin, Rutherfordstrasse 2, 12489 Berlin, Germany. e-mail: heinz-wilhelm.huebers@dlr.de

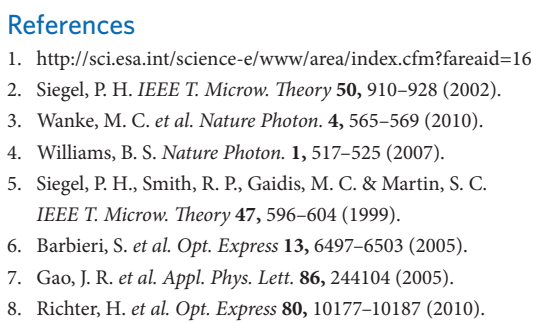

\section{Entangled photons report for duty}

Entangled photons are a key ingredient in optical quantum technologies, but researchers have so far been unable to produce a single pair of entangled photons. Now, two groups from China and Austria independently report just that, with a technique that avoids the need to infer entanglement from detection signatures.

\section{Pieter Kok}

E ntanglement is arguably the most important ingredient for quantum information processing ${ }^{1}$. Without it there would be no quantum teleportation, quantum computing or long-range quantum communication. Optical quantum information processing in particular requires a source that can produce entangled photon pairs on demand. However, the most widely used source of entangled photons spontaneous parametric down-conversion in a nonlinear optical crystal - does not actually produce entangled photons. What is actually produced is a weak signal consisting of a superposition of different numbers of entangled photon pairs, with the largest contribution coming from the possibility that no photons have been produced. As 